\title{
Inclusão do cuidado com a saúde das pessoas com deficiência nos currículos de medicina do Brasil
}

\author{
Inclusion of health care for people with disabilities in Brazilian undergraduate medical education
}

\author{
Reginaldo Antônio de Oliveira Freitas Júnior ${ }^{1,2}$ (D) $\mid$ reginaldo@isd.org.br

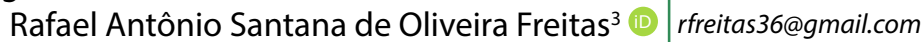 \\ Marcelo Pacheco Carvalho' (1) marcelo.carvalho@isd.org.br \\ Samantha Santos de Albuquerque Maranhão' (D) samantha.maranhao@isd.org.br \\ Lilian Lira Lisboa ${ }^{1,2}$ (D) lilian.lisboa@isd.org.br \\ George Dantas de Azevedo² (D) georgedantas.faimer@gmail.com
}

\section{RESUMO}

Introdução: No Brasil, estima-se que haja 45,6 milhões de pessoas com alguma deficiência. As disparidades de saúde enfrentadas pelas pessoas com deficiência (PCD) decorrem, entre outros fatores, do acesso inadequado aos cuidados de saúde e da formação deficiente dos profissionais para lidar com essas situações.

Objetivo: Este estudo teve como objetivo identificar a presença de aspectos relacionados com a atenção à saúde das PCD nos currículos dos cursos de Medicina do Brasil.

Método: Foi realizada análise documental dos projetos pedagógicos dos cursos, dos currículos, das ementas de componentes curriculares e dos conteúdos programáticos, incluindo pesquisas em sites de 328 cursos de Medicina (42\% públicos e 58\% privados) autorizados pelo Ministério da Educação. As Diretrizes Curriculares Nacionais (DCN) do Curso de Graduação em Medicina foram adotadas como referencial teórico. A classificação considerada foi: classe I - atendimento mínimo do projeto pedagógico às recomendações sobre o cuidado para PCD, pela estrita transcrição do texto das DCN, e classe II - inclusão nas ementas e nos conteúdos programáticos dos componentes curriculares de atividades voltadas ao desenvolvimento de competências clínicas especificamente relacionadas ao cuidado destinado à saúde das PCD, incluindo comunicação, exame clínico e aspectos éticos. Resultado: Documentos de 171 cursos estavam disponíveis para análise adequada. Desse total, em 89 cursos (52\%) foi identificada a inclusão de aspectos relacionados ao cuidado com PCD, sendo mais prevalente nos cursos públicos ( $\mathrm{n}=56 ; 62,9 \%$ ). Em 50 ( $29,2 \%)$ cursos, observouse a inclusão do ensino da Língua Brasileira de Sinais (Libras). Os conteúdos curriculares identificados foram predominantemente focados na classe I ( $\mathrm{n}=80 ; 89,9 \%)$, com absoluta falta de descrição das estratégias processuais para promover o desenvolvimento de competências clínicas relacionadas ao cuidado para PCD.

Conclusão: No contexto brasileiro, os dados apontam para uma situação dramática no que se refere à invisibilidade das questões relativas às PCD na formação médica e para a necessidade de desenvolvimento e implementação de estratégias educacionais especificamente voltadas para o cuidado com as PCD nos currículos médicos. O estudo reforça o papel essencial do cuidado competente destinado à saúde das PCD como estratégia que visa à mitigação das iniquidades de saúde enfrentadas por essas pessoas.

Palavras-chave: Pessoas com Deficiência; Educação Médica; Currículo; Escolas Médicas.

\begin{abstract}
Introduction: In Brazil, official data estimate there are 45.6 million people with some type of disability and legislation establishes that medical schools should prepare future professionals for the essential care for people with disabilities (PWD). Health disparities faced by PWD are due, among other factors, to inadequate access to healthcare and poor training of professionals to deal with these situations.

Objective: To identify the presence of aspects related to PWD healthcare in the curricula of medical courses in Brazil.

Method: A documentary analysis of curricula and syllabuses was performed and included research in websites of 328 medical courses (42\% public and $58 \%$ private) authorized by the Brazilian Ministry of Education. The Brazilian National Curricular Guidelines (NCG) were adopted as a theoretical framework (Brazil, 2014). The classification considered was: Class I - minimum attendance of the pedagogical project to the recommendations on care for PWD, due to the strict transcription of the NCG text and Class II - inclusion in the syllabus and programmatic content of the reference curricular components to the development of specifically related clinical skills to the health care of the PWD, including communication, clinical examination skills and ethical aspects.

Results: Documents from 171 courses were available for analysis and the inclusion of aspects related to PWD healthcare was identified in 89 courses (52\%). This inclusion was more prevalent in public courses $(n=56 ; 62,9 \%)$ than in private ones. The inclusion of the teaching of the Brazilian Sign Language (Libras) was observed In 50 courses (29.2\%). The curricular contents were predominantly focused on the Class I ( $n=80 ; 89,9 \%)$ with an absolute lack of description of the procedural strategies to promote the development of clinical competencies related to PWD care.
\end{abstract}

Conclusion: Our study reinforces the need to improve this approach in medical school curricula since providers can play an essential role in mitigating health disparities faced by PWD through competent care. In the Brazilian context, our data point out to a dramatic situation consistent with the invisibility of PWD issues; a need for the development and implementation of PWD-specific educational curricula.

Keywords: Disabled Persons; Medical Education; Curriculum; Medical Schools.

${ }^{1}$ Instituto de Ensino e Pesquisa Alberto Santos Dumont, Macaíba, Rio Grande do Norte, Brasil.

${ }^{2}$ Universidade Federal do Rio Grande do Norte, Natal, Rio Grande do Norte, Brasil.

${ }^{3}$ Faculdade de Ciências Médicas da Santa Casa de São Paulo, São Paulo, São Paulo, Brasil.

Editora-chefe: Rosiane Viana Zuza Diniz. Editor associado: Kristopherson Lustosa Augusto.

Recebido em 03/03/21; Aceito em 07/06/21. Avaliado pelo processo de double blind review. 


\section{INTRODUÇÃO}

A Organização Mundial da Saúde estima que 15\% da população mundial apresente incapacidades ${ }^{1}$. No Brasil, segundo o Censo do Instituto Brasileiro de Geografia e Estatística (IBGE) de 2010, 45,6 milhões de pessoas declararam possuir algum tipo de deficiência, o que representa 23,9\% da população residente no país ${ }^{2}$. Esses números expressam a importância que a atenção às necessidades de saúde dessa população específica deve assumir no contexto da efetividade dos sistemas de saúde.

O Plano Nacional dos Direitos da Pessoa com Deficiência - Viver sem Limite $^{3}$ ressalta o compromisso do Brasil com as prerrogativas da Convenção sobre os Direitos das Pessoas com Deficiência da Organização das Nações Unidas $(\mathrm{ONU})^{4}$, ratificada com equivalência de emenda constitucional. Por sua vez, a Política Nacional de Saúde das Pessoas com Deficiência propõe, entre outras diretrizes, a necessidade de capacitação de recursos humanos e aponta como prioritária a disponibilidade de profissionais de saúde capacitados para o desenvolvimento das ações dela decorrentes. Ainda segundo tais diretrizes, os programas de formação e treinamento desses profissionais devem ser promovidos por diferentes órgãos envolvidos com a questão, nos níveis federal, estadual e municipal, incluindo o engajamento das instituições públicas não estatais. Adicionalmente, ações integradas do Ministério da Educação (MEC) e de instituições de ensino superior (IES) devem ser promovidas, tendo em vista a necessidade de que sejam incorporados conteúdos e disciplinas que abordem reabilitação e atenção à saúde das pessoas com deficiência (PCD) nos currículos de graduação da área da saúde ${ }^{5}$.

A parceria entre os Ministérios da Saúde e da Educação busca a difusão, em parceria com as instituições de ensino e a comunidade escolar, de informações relacionadas às deficiências, à prevenção e à limitação das incapacidades de modo a contribuir para a qualidade de vida desse segmento populacional. Isso remete à necessidade de adequação dos currículos dos cursos de graduação da área da saúde para a inclusão de conteúdos relacionados à saúde das PCD, bem como o desenvolvimento de metodologias e materiais didáticopedagógicos, visando ao atendimento das diretrizes fixadas na Política Nacional de Saúde das Pessoas com Deficiência 5 .

O Brasil tem avançado na implementação dos apoios necessários ao pleno e efetivo exercício do protagonismo e da capacidade legal por todas as PCD. Entretanto, para que as prerrogativas da Convenção da ONU verdadeiramente aconteçam na vida dessas pessoas, há que se concretizar a ampla articulação de políticas governamentais de acesso à educação, inclusão social, atenção à saúde e promoção de acessibilidade. $A$ formação dos profissionais de saúde não pode estar dissociada dessa articulação social e precisa responder a suas demandas específicas. Logo, a perspectiva da integralidade na atenção à saúde das $P C D$, para além da assistência específica à sua condição, deve também incluir a assistência às comorbidades e aos agravos potencializados pelos mais diversos determinantes sociais do processo saúde-doença.

Será que os pressupostos teóricos relacionados à atenção à saúde das PCD expressos nas Diretrizes Curriculares Nacionais (DCN) do Curso de Graduação em Medicina ${ }^{6}$ tornaram-se realidade nas escolas médicas brasileiras? Essa é a pergunta que instigou a realização da presente pesquisa, cujo objetivo foi identificar a presença de aspectos relacionados com a atenção à saúde das PCD nos currículos dos cursos de Medicina do Brasil.

\section{MÉTODO}

Realizou-se um estudo descritivo, cujo percurso metodológico envolveu a análise documental de projetos pedagógicos de cursos de Medicina ofertados por IES do Brasil, além de ementas e conteúdos programáticos de disciplinas e módulos, com vistas a identificar se os referidos documentos institucionais expressam aspectos relacionados às necessidades de saúde das PCD.

O referencial teórico utilizado para a análise dos dados da pesquisa foi a normativa instituída pelas $\mathrm{DCN}^{6}$, nos termos da Resolução n 3, de 20 de junho de 2014, da Câmara de Educação Superior (CES) e do Conselho Nacional de Educação (CNE) do MEC, que estabelece a necessidade de inclusão do cuidado destinado à saúde das PCD na formação dos médicos no Brasil. Como critérios de inclusão foram considerados os cursos de Medicina constantes no Cadastro e-MEC de instituições e cursos de educação superior ${ }^{7}$, base de dados oficial e única de informações relativas às IES e aos cursos de graduação do sistema federal de ensino, até a data de 30 de novembro de 2018. Os dados do Cadastro e-MEC estão em conformidade com os atos autorizativos das instituições e dos cursos de educação superior, editados com base nos processos regulatórios competentes, nos termos da Portaria Normativa do MEC n॰ 40/2007 .

Além do registro no Cadastro e-MEC, outro critério de inclusão considerado foi a disponibilidade dos projetos pedagógicos dos cursos (PPC), das ementas e dos conteúdos programáticos de disciplinas e módulos nas páginas eletrônicas públicas das IES. Excluíram-se da amostra os cursos cujas instituições não disponibilizaram os referidos documentos para acesso público. A partir dessas informações oficiais, buscaramse informações detalhadas sobre os diversos cursos, com base em consulta aos sítios eletrônicos disponíveis na internet, utilizando formulário de pesquisa previamente definido. 
Uma vez constituído o conjunto dos documentos para estudo, partiu-se para um processo de análise, que incluiu três etapas: 1. pré-análise, 2. análise e 3. classificação. Durante a pré-análise, realizou-se uma busca computadorizada de termos presentes nos documentos oficiais dos cursos que remetessem à saúde das $\mathrm{PCD}$. Utilizaram-se como indicadores de busca as seguintes palavras-chave: "pessoa com deficiência", "PCD", "deficiência", "deficiente", "deficiência física", "deficiente físico", "paraplégico", "paraplégica", "paraplegia", "tetraplégico", "tetraplégica”, "tetraplegia", "deficiência intelectual", "deficiente intelectual", "déficit cognitivo", "retardo mental", "paralisia cerebral", "autismo", "autista", "deficiência visual", "cego", "cega", "cegueira", "deficiência auditiva", "surdo", "surda", "surdez", "mudo", "muda", “mudez", "surdo-mudo", "surda-muda", "afásico", "afásica", "afasia", "Libras", "pessoa com necessidades especiais", "acessibilidade", "competências clínicas", "atendimento", "atender", "anamnese", "entrevista clínica”, "exame", "exame físico”, "exame clínico", "inspeção", "palpação", "apalpação", "percussão", "ausculta". Essa etapa de busca computadorizada dos termos foi complementada por uma leitura detalhada dos parágrafos e das seções cujo emprego das palavras-chave fazia referência à saúde das PCD. Essa primeira leitura, menos sistemática, permitiu a familiarização inicial com os documentos e, ao mesmo tempo, o reconhecimento e a identificação das unidades de registro, ou seja, dos trechos que veiculavam conteúdos relacionados com a atenção à saúde das PCD, bem como com a descrição do desenvolvimento de competências clínicas, como a realização da anamnese e do exame físico.

As etapas 2 e 3 de análise propriamente dita do material foram realizadas de forma concomitante e permitiram classificar as escolas médicas pela busca de características compartilhadas pelas unidades de registro, seja mediante critérios semânticos (isto é, busca da presença de um mesmo significado em um determinado contexto) e/ou sintáticos (como a presença compartilhada de signos linguísticos precisos). Nessa fase da análise, os documentos foram examinados integralmente, localizando as unidades de registro e transcrevendo-se em uma tabela tanto essas unidades quanto as unidades de contexto que correspondiam a trechos mais amplos do texto nos quais estavam contidas as unidades de registro. A partir das unidades de contexto, foi possível analisar de modo mais preciso o significado atribuído ao termo ou conceito de interesse utilizado em cada unidade de registro.

Tomando-se por base o referencial teórico das $\mathrm{DCN}^{6}$ de Medicina, definiram-se duas classes de cursos médicos, a saber:

- Classe I: Atendimento mínimo do PPC às recomendações sobre o cuidado para PCD.

- Classe II: Inclusão nas ementas e nos conteúdos programáticos dos componentes curriculares de atividades voltadas ao desenvolvimento de competências clínicas especificamente relacionadas ao cuidado destinado à saúde das PCD.

O Quadro 1 apresenta o detalhamento dos critérios e o referencial para construção dessas classes, a partir da contextualização de como o tema pesquisado emergiu nos documentos oficiais dos cursos de Medicina.

O presente estudo utilizou exclusivamente dados secundários disponíveis em documentos de acesso público, sem identificação dos sujeitos, no caso dos cursos de Medicina do Brasil, o que está de acordo com os preceitos éticos estabelecidos na Resolução n 196, de 10 de outubro de 1996, do Conselho Nacional de Saúde do Ministério da Saúde.

\section{RESULTADOS}

Do total de 328 cursos de Medicina (42\% públicos e 58\% privados) constantes no Cadastro e-MEC de instituições e cursos de educação superior ${ }^{7}$, os documentos de 171 (52,1\%) cursos estavam disponíveis para análise adequada, incluindo PPC, currículos, ementas e conteúdos programáticos das disciplinas ou dos módulos, e foram, portanto, incluídos no estudo.

Entre os cursos incluídos no estudo, identificou-se, em 89 (52\%), a inclusão de aspectos relacionados ao cuidado com PCD nos documentos publicizados, e, em 50 (29,2\%) cursos, observou-se a inclusão do ensino da Língua Brasileira de Sinais (Libras), e em 18 (10,5\%) essa foi a única referência relacionada às PCD encontrada nesses documentos. A inclusão foi mais prevalente nos cursos públicos ( $n=56 ; 62,9 \%$ ) que nos privados ( $\mathrm{n}=33 ; 37,1 \%$ ). Dentre os cursos que incluíram aspectos relacionados ao cuidado com PCD em seus currículos, 63 (70,8\%) foram abertos após a vigência das DCN, ou seja, a partir de 2014. A distribuição desses cursos por região do país está apresentada na Tabela 1.

A Figura 1 ilustra que a absoluta maioria dos cursos que incluiu aspectos relacionados ao cuidado destinado às PCD em seus currículos $(80 ; 89,9 \%)$ assim o fez predominantemente focada na classe I e, mais especificamente, reproduziu, em seus documentos oficiais, o conteúdo textual que consta nas DCN. Tornou-se evidente a carência de descrição das estratégias processuais para promover o desenvolvimento de competências clínicas relacionadas ao cuidado com PCD, no presente estudo nominadas como classe II, incluindo comunicação, exame clínico e aspectos éticos. Dos nove cursos cujos currículos foram enquadrados na Classe II, oito eram públicos e oito foram abertos após a publicação das DCN vigentes. Tais referências limitaram-se à transcrição de fragmentos textuais do artigo 12, inciso II, alínea "c" das DCN, a saber: 
[...] postura ética, respeitosa e destreza técnica na inspeção, apalpação, ausculta e percussão, com precisão na aplicação das manobras e procedimentos do exame físico geral e específico, considerando a história clínica, a diversidade étnico-racial, de gênero, de orientação sexual, linguístico-cultural e de pessoas com deficiência.

A Tabela 2 apresenta a distribuição dos cursos de Medicina que incluíram aspectos relacionados ao cuidado com as PCD em seus currículos, segundo as classes de análise classes I e II - e tipo de financiamento, se públicos ou privados, e conforme abertura após a publicação das DCN vigentes, ou seja, se antes ou depois de 2014.
Tabela 1. Distribuição dos cursos de Medicina, públicos e privados, que incluíram aspectos relacionados ao cuidado destinado às pessoas com deficiência em seus currículos, segundo as grandes regiões do Brasil.

\begin{tabular}{cccc}
\hline Região & Públicos & Privados & Total \\
\hline Norte & $5(5,6 \%)$ & $6(6,7 \%)$ & $11(12,4 \%)$ \\
Nordeste & $21(23,6 \%)$ & $4(4,5 \%)$ & $25(28,1 \%)$ \\
Centro-Oeste & $6(6,7 \%)$ & $3(3,4 \%)$ & $9(10,1 \%)$ \\
Sudeste & $15(16,9 \%)$ & $15(16,9 \%)$ & $30(33,7 \%)$ \\
Sul & $9(10,1 \%)$ & $5(5,6 \%)$ & $14(15,7 \%)$ \\
\hline Total & $56(62,9 \%)$ & $33(37,1 \%)$ & $89(100,0 \%)$ \\
\hline
\end{tabular}

Fonte: Elaborada pelos autores.

Quadro 1. Descrição da classificação de análise utilizada, tendo como referência as Diretrizes Curriculares Nacionais do Curso de Graduação em Medicina ${ }^{6 .}$

\title{
Classe I - Atendimento mínimo do PPC às recomendações sobre o cuidado para PCD
}

Critério utilizado: Cursos cujas unidades de registro enquadravam-se nas recomendações das DCN relacionadas à "inserção da temática da diversidade na formação em Medicina com vistas à promoção da equidade em saúde"; estrita transcrição no projeto pedagógico do texto recomendado pelas DCN.

Referencial nas DCN ${ }^{6}$ :

\author{
CAPÍTULO I \\ DAS DIRETRIZES \\ Seção I \\ Da Atenção à Saúde
}

Art. 5․ Na Atenção à Saúde, o graduando será formado para considerar sempre as dimensões da diversidade biológica [...] e demais aspectos que compõem o espectro da diversidade humana [...] no sentido de concretizar: [...]

X - promoção da equidade no cuidado adequado e eficiente das pessoas com deficiência [...].

\section{CAPÍTULO III \\ DOS CONTEÚDOS CURRICULARES E DO PROJETO PEDAGÓGICO DO CURSO DE GRADUAÇÃO EM MEDICINA}

Art. 23. Os conteúdos fundamentais (...) devem estar relacionados com todo o processo saúde-doença do cidadão, da família e da comunidade, [...] proporcionando a integralidade das ações do cuidar em saúde, contemplando: [...]

VII - [...] temas transversais no currículo que envolvam conhecimentos, vivências e reflexões sistematizadas acerca dos direitos humanos e de pessoas com deficiência [...].

Classe II - Inclusão nas ementas e nos conteúdos programáticos dos componentes curriculares de atividades voltadas ao desenvolvimento de competências clínicas especificamente relacionadas ao cuidado destinado à saúde das PCD

Critério utilizado: Cursos cujas unidades de análise ampliam os critérios descritos para a classe I, com menção ao desenvolvimento de competências clínicas especificamente relacionadas ao cuidado destinado à saúde das $P C D$, incluindo comunicação, exame clínico e aspectos éticos, além de descrição nas ementas e nos conteúdos programáticos dos componentes curriculares de habilidades clínicas e assistência ao paciente.

\section{Referencial nas DCN6:}

\section{CAPÍTULO II}

DAS ÁREAS DE COMPETÊNCIA DA PRÁTICA MÉDICA [...]

Subseção I

Da Atenção às Necessidades Individuais de Saúde [...]

Art. 12. [...]

II - Realização do Exame Físico: [...]

c) postura ética, respeitosa e destreza técnica na inspeção, apalpação, ausculta e percussão, com precisão na aplicação das manobras e procedimentos do exame físico geral e específico, considerando a história clínica, a diversidade étnico-racial, de gênero, de orientação sexual, linguísticocultural e de pessoas com deficiência [...].

Fonte: Elaborado pelos autores. 
Figura 1. Distribuição dos cursos de Medicina que incluíram aspectos relacionados ao cuidado destinado às pessoas com deficiência em seus currículos, segundo a classificação adotada no estudo.

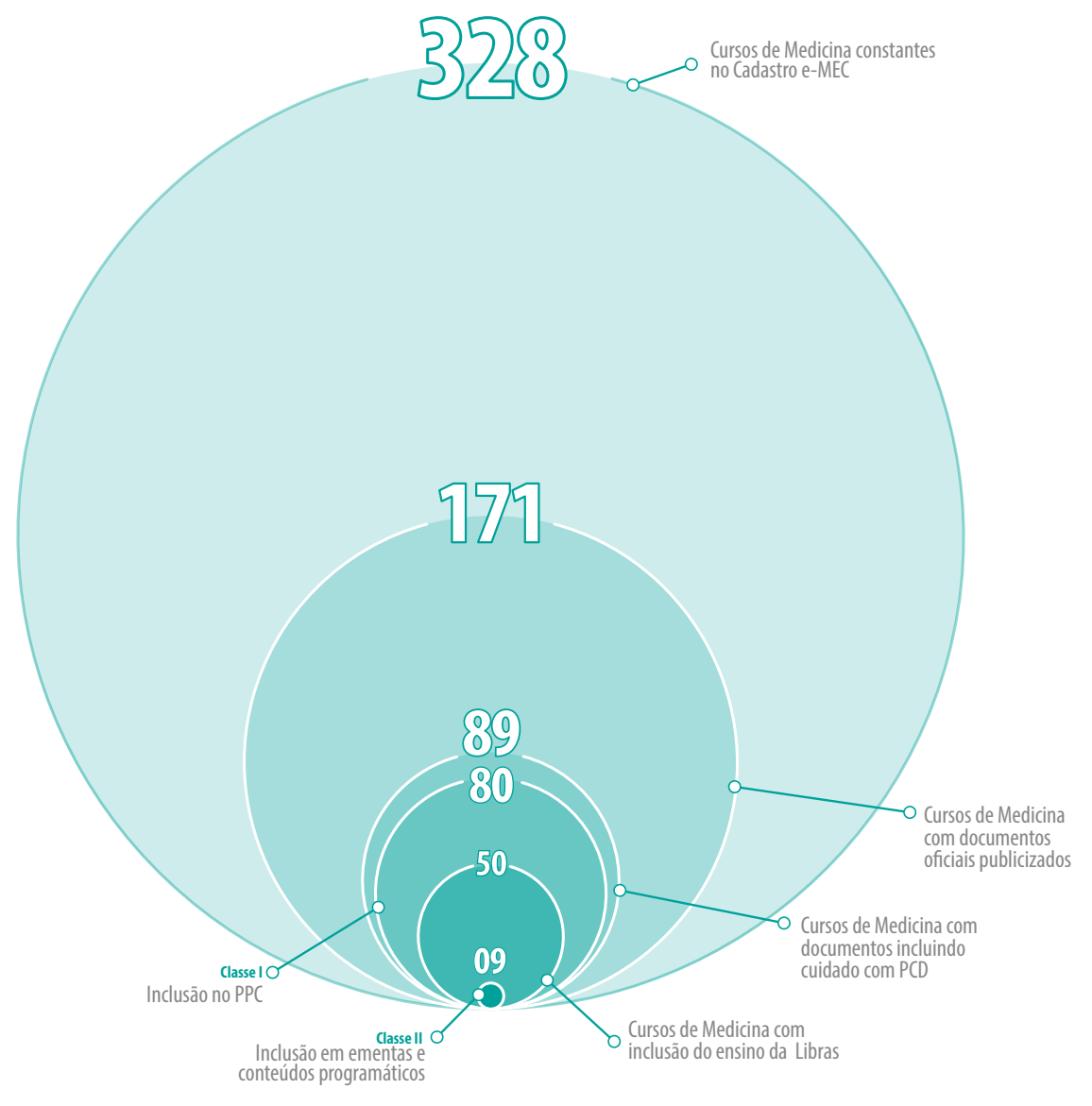

Fonte: Elaborada pelos autores.

Tabela 2. Distribuição dos cursos de Medicina que incluíram aspectos relacionados ao cuidado destinado às pessoas com deficiência em seus currículos, segundo as classes de análise, o tipo de financiamento (públicos ou privados) e a época de abertura.

\begin{tabular}{ccccc}
\hline & Públicos & Privados & Abertura antes de 2014 & Abertura depois de 2014 \\
\hline $\begin{array}{c}\text { Classe I } \\
\text { Subtotal = } 80(89,9 \%)\end{array}$ & $48(60 \%)$ & $32(40 \%)$ & $25(31,2 \%)$ & $55(68,8 \%)$ \\
$\begin{array}{c}\text { Classe II } \\
\text { Subtotal = 09 }(10,1 \%)\end{array}$ & $8(88,9 \%)$ & $1(11,1 \%)$ & $1(11,1 \%)$ & $8(88,9 \%)$ \\
\hline Total = 89 (100\%) & $56(62,9 \%)^{\mathrm{a}}$ & $33(37,1 \%)^{\mathrm{a}}$ & $26(29,2 \%)^{\mathrm{a}}$ & $63(70,8 \%)^{\mathrm{a}}$ \\
\hline
\end{tabular}

Classe I - Atendimento mínimo do PPC às recomendações sobre o cuidado para PCD; classe II - Inclusão nas ementas e nos conteúdos programáticos dos componentes curriculares de atividades voltadas ao desenvolvimento de competências clínicas especificamente relacionadas ao cuidado destinado à saúde das PCD.

a Percentuais expressos em relação ao total de cursos (89/100\%).

Fonte: Elaborada pelos autores.

\section{DISCUSSÃO}

Os estudantes de Medicina relataram que se sentem desconfortáveis ao interagirem com PCD, e estudantes de Medicina, residentes e médicos em atividade demonstraram deficiências no conhecimento prático até mesmo das formas mais comuns de deficiência9.

A questão central a ser discutida pelas escolas médicas brasileiras é como concretizar o cuidado integral destinado à saúde das PCD no Brasil se o desenvolvimento das competências clínicas para o exercício desse cuidado não vem sendo estimulado durante a formação dos médicos brasileiros. Os resultados observados no presente estudo denunciam essa realidade quando identificam a ausência de menção às PCD nos currículos de $48 \%$ (82) dos 171 cursos cujos documentos oficiais estavam disponíveis para consulta pública. Não menos preocupante é a constatação de que os outros $52 \%$ (89) desses 
cursos, em sua quase totalidade, se limitam à transcrição dos fragmentos textuais das DCN relacionados com a atenção à saúde das PCD, e somente 10,1\% (nove) desses 89 cursos, de alguma forma, incluíram aspectos relacionados à anamnese, ao exame físico ou aos aspectos éticos no cuidado com a saúde das PCD em seus componentes curriculares. Ambos os achados representam desafios a serem superados pela formação médica no país, na medida em que a importância ora dada à saúde das PCD pelas escolas médicas brasileiras não parece ser capaz de garantir a vivência de experiências formativas que habilitem, ou ao menos estimulem, os estudantes de Medicina para o cuidado com a saúde das PCD.

Na contemporaneidade, esse é um importante desafio para todas as escolas de Medicina, em todo o mundo. Nos Estados Unidos, as PCD representam 22,2\% da população, e estima-se que todos os médicos atendam PCD em sua prática clínica. Contudo, estudos apontam que as PCD são inadequadamente atendidas pelo sistema de saúde, sendo apontado como uma das causas prováveis o limitado treinamento dos profissionais de saúde no cuidado destinado à saúde das PCD. Nesse sentido, Ankam et al. $^{10}$ destacam a necessidade de introduzir nos currículos objetivos de aprendizagem especificamente relacionados à saúde das $P C D$, com vistas à capacitação dos futuros médicos para o cuidado adequado destinado essa população.

As escolas médicas representam espaços de grande importância para a produção dos conhecimentos em saúde, e importância equivalente deve ser atribuída a esses potentes espaços de formação profissional como agentes catalisadores da concretização das políticas públicas em saúde. No Brasil, há uma expressa garantia constitucional que confere ao Sistema Único de Saúde (SUS) a competência de, entre outras atribuições, ordenar a formação de recursos humanos na área de saúde ${ }^{11}$. No contexto do cuidado com a saúde das PCD, a articulação entre as escolas médicas e o SUS assume dimensão muito especial diante da premente necessidade de concretização da Política Nacional de Saúde das Pessoas com Deficiência, do Plano Nacional dos Direitos da Pessoa com Deficiência - Viver sem Limite e do fortalecimento da Rede de Cuidados à Pessoa com Deficiência.

Ciuffo et al. ${ }^{11}$ reforçam que reconhecer o SUS como ordenador da formação exige uma profunda reestruturação nos currículos de Medicina, especificamente, a ser realizada por meio de sucessivas e permanentes inovações curriculares, orientadas pelo princípio da integralidade - noção complexa que articula concepções e práticas de saúde - e pela revisão do processo de trabalho e de gestão em saúde. Para isso, fazem-se necessárias as rupturas do paradigma biomédico, que ainda sustenta as práticas em saúde e grande parte dos currículos de Medicina no Brasil; e da concepção simplista de que "ter saúde é não ter doença", o que implica retirar o foco das ações assistencialistas e entender que o cuidado, e não mais a assistência, deve conjugar ações de prevenção de doenças, promoção da saúde, além da cura e da reabilitação - todas exigindo pluralidade de saberes -, e o trabalho em equipe interdisciplinar e multiprofissional, preferencialmente em rede. As autoras destacam que muitas escolas ainda refletem e reproduzem a lógica fragmentada própria do cientificismo, deixando de potencializar o que há de mais rico no campo da educação: as relações possíveis de ser desenvolvidas entre professor e estudante que, mais tarde, revertem-se nas relações estabelecidas entre os profissionais e seus pacientes, ou entre quem cuida e quem é cuidado. Retrato, embora parcial, do descaso com a área das relações interpessoais vem sendo expresso, entre outros resultados, nos baixos índices de resolutividade do SUS, na insatisfação dos usuários e de muitos profissionais dos serviços, e na utilização de procedimentos de alto custo, muitas vezes, desnecessários ${ }^{11}$.

Relatos de PCD denunciam uma realidade bem distante da atenção integral preconizada. Harrington et al. ${ }^{12}$ apontam que muitos serviços de atenção primária nos Estados Unidos não são estruturados para responderem integralmente às demandas de PCD no que se refere à acessibilidade física, comunicacional e mesmo de atitudes. Os autores identificam mais barreiras médicas (physician barriers) do que barreiras físicas (physical barriers) nos serviços e constatam que as PCD são mais sensíveis às barreiras de atitudes do que às físicas. Somente $2,67 \%$ dos sujeitos pesquisados relatam dificuldades de acesso ao consultório, mas $36,4 \%$ relatam que tiveram que ensinar ao seu médico sobre sua deficiência, saindo da consulta com a sensação de não terem suas necessidades totalmente atendidas.

Há falta de esclarecimento aos familiares sobre as possibilidades de desenvolvimento das PCD e formas de superar as dificuldades encontradas, ou de orientações à estimulação precoce. Os médicos desconhecem as particularidades que diferenciam um corpo com e sem deficiência, o que dificulta a redução de riscos de surgimento de problemas de saúde evitáveis ou de procedimentos inadequados, que podem até mesmo levar à morte do paciente, como a hiper-reflexia autonômica intercorrência frequente em pessoas tetraplégicas. É raro as PCD encontrarem médicos com capacidade técnica aliada à abertura para negociação quanto ao cuidado com a saúde e que se preocupem com questões relacionadas ao serviço, como redução do tempo e da energia despendidos pelas PCD para receber atendimento médico. Por isso, elas acabam tendo que escolher qual dessas capacidades consideram prioritária, no momento, em detrimento das demais ${ }^{13}$.

Na ginecologia e obstetrícia, como exemplo de uma das áreas prioritárias da formação médica como estágio 
curricular obrigatório, a perspectiva da saúde da mulher com deficiência se revela merecedora de grandes transformações para a garantia do cuidado efetivo. Cada vez mais, mulheres com lesão medular optam por engravidar, demandando dos profissionais preparo no acompanhamento de uma gravidez com risco de trombose, infecção urinária de repetição e úlceras de pressão, à medida que a gestante ganha peso e hiper-reflexia autonômica ${ }^{13}$. Nicolau ${ }^{14}$ e Nicolau et al. ${ }^{15}$ enfatizam que mulheres com deficiência contam com ações inexpressivas nos serviços de atenção básica à saúde, que, embora historicamente privilegiem a clientela feminina, pouco reconhecem os aspectos relativos aos direitos sexuais e reprodutivos e à dupla vulnerabilidade que as acometem por serem mulheres e PCD. Algumas mulheres enfrentam preconceitos para viver a sexualidade e a maternidade, na medida em que habitam um corpo que destoa dos padrões estéticos vigentes e enfrentam a descrença da sociedade de que possam corresponder às expectativas de gênero, como assumir os papéis de cuidadora, esposa e mãe ${ }^{15}$.

Gomes et al. ${ }^{16}$, em estudo observacional do tipo transversal e descritivo, entrevistaram 101 médicos de 24 especialidades diferentes e perguntaram se o médico já havia atendido um paciente surdo, se o paciente estava acompanhado, qual sentimento percebido na consulta (ocorrido ou prospectivo), se o médico tinha conhecimento de Libras e se via o conhecimento de Libras como importante para sua prática médica. Nessa casuística, 92,1\% dos médicos entrevistados afirmaram já ter atendido pacientes surdos, e 76,2\% consideraram o conhecimento de Libras importante para sua prática médica. Apesar da expressiva proporção, apenas um médico dos 101 entrevistados declarou ter conhecimento básico de Libras, e os sentimentos predominantes entre os médicos entrevistados foram incerteza e desconforto durante o atendimento ${ }^{16}$.

As barreiras de comunicação representam verdadeiro óbice à efetividade do atendimento, à formação de vínculo e ao sucesso das orientações para tratamento, comprometendo a segurança do usuário. No cuidado destinado à saúde da pessoa surda, tais barreiras assumem potencial ainda mais danoso e devem ser superadas como um problema sociocultural. De acordo com os dados do IBGE colhidos em 2010, das 45.606 .048 pessoas no Brasil que têm algum tipo de deficiência, 7,6\% delas são totalmente surdas, ou seja $1,2 \%$ da população brasileira ${ }^{2}$. No presente estudo, a oferta da Libras como componente curricular, independentemente de ser ele obrigatório ou optativo, foi observada em menos de um terço ( $n=50 ; 29,2 \%$ ) dos cursos cujos currículos estavam disponíveis para avaliação, sendo a única referência ao cuidado com a saúde das PCD em 18 (10,5\%) deles. A Libras é um sistema linguístico tão complexo quanto a linguagem falada e é uma importante ferramenta para os profissionais de saúde no âmbito de diagnóstico e tratamento efetivo, estando diretamente relacionada ao bom atendimento do paciente. A percepção de que o paciente surdo merece acesso e tratamento como qualquer outro cidadão transparece nas diversas medidas legislativas, como a Lei Federal $n^{\circ} 10.436 / 2002$, que prevê a garantia do atendimento e tratamento adequado às pessoas com deficiências auditivas por parte de instituições públicas e concessionárias de serviços públicos na área da saúde; a Lei $n^{\circ}$ 10.098/2000 ou Lei de Acessibilidade, que implementa a formação de profissionais intérpretes no artigo 18; e o Decreto $n^{\circ} 5.626 / 2005$, que regulamenta que, em uma unidade de saúde, pelo menos $5 \%$ dos funcionários saibam Libras. Entretanto, apesar de contemplada pela legislação, a assistência ao surdo mostra-se precária. A comunicação adequada e a compreensão entre surdo e ouvinte são fatores primordiais da inclusão, a qual dificilmente pode ocorrer com tão poucos ouvintes conhecedores de Libras. Embora o ensino de Libras seja oferecido facultativamente para a maioria dos cursos no ensino superior desde os últimos dez anos, vemos pouca expressividade desse conhecimento entre os profissionais de saúde ${ }^{16}$.

A verdadeira inclusão das PCD no ensino médico deve se dar nas várias disciplinas ao longo do curso, incluindo as das áreas clínica e cirúrgica. Discutir temas ligados à deficiência em uma disciplina optativa ou em único momento no curso pode ser um primeiro passo, mas não deve ser o único. $\mathrm{O}$ cuidado no pré-natal e parto de gestantes com deficiência, por exemplo, deve ser ensinado na disciplina de Obstetrícia; nas aulas de Semiologia, os alunos devem aprender como se comunicar com pessoas surdas ou com dificuldades na fala, ou como reconhecer uma doença com base em sinais e sintomas que se apresentam de forma distinta em pessoas com lesão medular. Mais ainda, é importante que a inclusão da atenção à saúde das PCD seja planejada atendendo a uma lógica e que os professores/médicos tenham sua prática pautada pelo modelo biopsicossocial de perceber a deficiência. Por último, é indispensável o contato direto com PCD no papel de educadores, participando em conversas, como palestrantes ou pacientes padronizados ${ }^{17}$. A exposição dos estudantes de Medicina a um currículo longitudinal para cuidar de PCD levou a uma melhora significativa em vários fatores relacionados ao conforto e às atitudes 9 .

Bosques et al. ${ }^{18}$ realizaram um estudo de intervenção envolvendo oferta de um curso eletivo para estudantes de Medicina do terceiro ou quarto anos, em que eles tinham a oportunidade de desenvolver o cuidado para pacientes com agravos neurológicos e musculoesqueléticos, e participar de sessões de reabilitação. Além dos métodos pedagógicos tradicionais, o curso incluiu metodologias centradas nos 
estudantes, com destaque para a experiência prática no manejo da cadeira de rodas. Os resultados demonstraram ganho de conhecimento significativo acerca da terminologia relacionada à saúde das $\mathrm{PCD}$, quando comparados com os períodos pré e pós-intervenção. Adicionalmente, os estudantes de Medicina tiveram ganho cognitivo sobre a legislação relacionada e sobre recursos que podem ser utilizados na atenção às $P C D$, com vistas a reduzir as barreiras para o cuidado adequado. Como conclusão deste estudo, sugere-se que a inclusão dos tópicos relativos à saúde das $P C D$ nos currículos médicos, de forma longitudinal, é importante para preencher a lacuna atualmente existente no acesso aos cuidados de saúde para as PCD.

Para a efetivação do cuidado integral com a saúde das PCD no sistema de saúde, impõe-se à formação em saúde a ruptura do paradigma da concepção de saúde dessas pessoas centrada na deficiência como doença, na qual essa população é sempre vista pelo profissional de saúde como alguém doente. Nesse caso, deve-se considerar a concepção da deficiência como diferença, já que a PCD é alguém que apresenta limitações maiores ou menores, o que dependerá do nível de acessibilidade arquitetônica/urbanística, comunicacional, instrumental, metodológica, programática ou atitudinal ${ }^{19}$. Muitas PCD, particularmente quando a situação de saúde delasé congênita ou de longo tempo, não necessariamente percebem a própria deficiência como um problema ou uma patologia. Ter uma deficiência não é incompatível com estar saudável, e não deve ser presumido que a queixa da consulta esteja sempre relacionada com a deficiência ${ }^{20}$. Para essa evolução na direção da integralidade do cuidado, os profissionais de saúde precisam perceber a pessoa além da deficiência e distinguir doença de deficiência, evitando medicalizar a deficiência, ou seja, declarar como objeto de tratamento uma variação que funcionalmente não gera danos, como o uso de hormônio do crescimento para "tratar" estatura muito baixa em crianças que não apresentam nenhuma patologia subjacente ${ }^{21}$.

Outro aspecto fundamental para que a formação médica efetivamente contribua para a redução das iniquidades em saúde enfrentadas pelas PCD é a integração entre a rede de atenção à saúde e as escolas médicas, no sentido de viabilizar cenários de prática que sejam capazes de garantir vivências catalisadoras do processo de ensino e aprendizagem nessa dimensão específica do cuidado. Sem ter experimentado ou vivenciado o cuidado com a saúde das PCD durante a formação, torna-se muito mais difícil ser efetivo nesse cuidado no exercício da profissão. Tais vivências devem oportunizar a relação dos conhecimentos teóricos e científicos com a realidade de vida das $P C D$, ressaltando as experiências concretas desses sujeitos como situações significativas de aprendizagem. Da mesma forma, os currículos, as disciplinas e os docentes devem contemplar o cuidado com a saúde das PCD para além da inclusão de conteúdos teóricos, priorizando práticas pedagógicas dinâmicas que integrem estudantes com PCD e reconheçam ambos como sujeitos ativos e protagonistas do processo de ensino e aprendizagem.

Nosso estudo tem algumas limitações potenciais. A técnica empregada para coleta de dados, que envolveu a pesquisa dos documentos públicos disponíveis nos sítios eletrônicos das IES, pode ter subestimado o número de cursos de Medicina que incluam aspectos relacionados à saúde das PCD em seus currículos. Isso pode ser especialmente importante quando se considera que um contingente significativo deescolas médicas brasileiras ainda não adota a cultura de disponibilizar PPC, ementas e conteúdos programáticos para acesso público. Todavia, a necessidade de atender a critérios regulatórios certamente influenciou o achado observado de muitos cursos simplesmente transcreverem as recomendações das DCN em seus documentos oficiais. Assim, o estudo não permite inferir que os estudantes desses cursos vivenciem experiências efetivamente formadoras para o cuidado destinado às PCD. Por fim, a pesquisa baseada em análise documental não é capaz de avaliar conhecimentos, habilidades e atitudes dos aprendizes; cabe, portanto, reforçar que o estudo foi desenhado para identificar a visibilidade da temática nos cursos de Medicina no Brasil. Pesquisas subsequentes são necessárias para avaliar a efetividade das estratégias didático-pedagógicas com vistas ao desenvolvimento de competências clínicas no cuidado com a saúde das PCD.

\section{CONSIDERAÇÕES FINAIS}

Nos currículos dos cursos brasileiros de Medicina, a inclusão de estratégias educacionais especificamente voltadas para o cuidado com a saúde das PCD ainda se mostra incipiente como mecanismo promotor da formação de profissionais de saúde requerida pela Política Nacional de Saúde das Pessoas com Deficiência. No Brasil, os dados obtidos nos documentos institucionais disponíveis nas páginas eletrônicas públicas das IES que oferecem cursos de Medicina apontam para uma situação dramática no que se refere à invisibilidade das questões relativas às PCD na formação médica e para a necessidade de desenvolvimento e implementação de estratégias educacionais especificamente voltadas para o cuidado destinado às PCD nos currículos médicos. O estudo reforça o papel essencial do cuidado competente com a saúde das PCD como estratégia de mitigação das iniquidades de saúde enfrentadas por essas pessoas.

\section{AGRADECIMENTOS}

Agradecemos ao Ministério da Educação e ao Instituto Santos Dumont os suportes financeiro e institucional, e a Fernando de 
Souza Silva, o design gráfico da ilustração sobre os resultados observados.

\section{CONTRIBUIÇÃO DOS AUTORES}

Reginaldo Antônio de Oliveira Freitas Júnior e George Dantas de Azevedo participaram da concepção e do delineamento do estudo, da análise e interpretação dos dados, da redação do artigo, da revisão crítica do conteúdo intelectual e da aprovação final da versão a ser publicada. Rafael Antônio Santana de Oliveira Freitas participou da coleta de dados, do desenvolvimento das ferramentas de análise documental, da análise e interpretação dos dados, e da redação do artigo e da aprovação final da versão a ser publicada. Marcelo Pacheco Carvalho participou da coordenação do processo de coleta de dados e do desenvolvimento das ferramentas de análise documental, da coleta e interpretação dos dados, e da redação do artigo e da aprovação final da versão a ser publicada. Samantha Santos de Albuquerque Maranhão e Lilian Lira Lisboa participaram da redação do artigo, da revisão crítica do conteúdo intelectual e da aprovação final da versão a ser publicada.

\section{CONFLITO DE INTERESSES}

Declaramos não haver conflito de interesses.

\section{FINANCIAMENTO}

Declaramos não haver financiamento.

\section{REFERÊNCIAS}

1. World Health Organization. World report on disability. Geneva:WHO; 2011 [access in 20 may 2019]. Available from: https://www.who.int/disabilities/ world_report/2011/report.pdf.

2. Instituto Brasileiro de Geografia e Estatística. Censo demográfico 2010. Características gerais da população, religião e pessoas com deficiência. Rio de Janeiro: IBGE; 2010 [access in 20 may 2019]. Available from: https:// biblioteca.ibge.gov.br/visualizacao/periodicos/94/cd_2010_religiao_ deficiencia.pdf.

3. Brasil. Plano Nacional dos Direitos da Pessoa com Deficiência - Viver sem Limite. Brasília: Secretaria Nacional de Promoção dos Direitos da Pessoa com Deficiência; 2013.

4. Organização das Nações Unidas. Convenção Internacional sobre os Direitos das Pessoas com Deficiência e seu Protocolo Facultativo. Nova York: ONU; 2007.

5. Brasil. Portaria $n^{\circ} 1.060$, de 5 de junho de 2002. Art. $1^{\circ}$ Aprovar, na forma do Anexo desta Portaria, a Política Nacional de Saúde da Pessoa Portadora de Deficiência. Diário Oficial da República Federativa do Brasil; 2002. Seção 1, p. 21.
6. Brasil. Resolução CNE/CES no 3, de 20 de junho de 2014. Institui Diretrizes Curriculares Nacionais do Curso de Graduação em Medicina e dá outras providências. Diário Oficial da República Federativa do Brasil; 2014.

7. Brasil. Portaria Normativa n 21 , de 21 de dezembro de 2017. Dispõe sobre o sistema e-MEC, sistema eletrônico de fluxo de trabalho e gerenciamento de informações relativas aos processos de regulação, avaliação e supervisão da educação no sistema federal de educação, e o Cadastro Nacional de Cursos e Instituições de Educação Superior - Cadastro e-MEC. Brasília: Ministério da Educação; 2017.

8. Brasil. Portaria Normativa MEC $n^{\circ} 40 / 2007$. Institui o e-MEC, sistema eletrônico de fluxo de trabalho e gerenciamento de informações relativas aos processos de regulação, avaliação e supervisão da educação superior no sistema federal de educação, e o Cadastro e-MEC de Instituições e Cursos Superiores e consolida disposições sobre indicadores de qualidade, banco de avaliadores (Basis) e o Exame Nacional de Desempenho de Estudantes (ENADE) e outras disposições. Brasília: Ministério da Educação; 2007.

9. Symons AB, Morley CP, McGuigan D, AkI EA. A curriculum on care for people with disabilities: effects on medical student self-reported attitudes and comfort level. Disabil Health J. 2014 Jan;7(1):95-88.

10. Ankam NS, Bosques G, Sauter C, Stiens S, Therattil M, Williams FH, et al. Competency-based curriculum development to meet the needs of people with disabilities: a call to action. Acad Med. 2019 June;94(6):781-8.

11. Ciuffo RS, Ribeiro VMB. Sistema Único de Saúde e a formação dos médicos: um diálogo possível? Interface Comun Saúde Educ. 2008;12(24):125-40.

12. Harrington AL, Hirst MA, Hammond FM, Norton HJ, Bocknek MD. Assessment of primary care services and perceived barriers to care in persons with disabilities. Am J Phys Med Rehabil. 2009;88(10):852-63.

13. Costa LSM. Educação médica e atenção integral à saúde da pessoa com deficiência. Rev Bras Educ Med. 2011;35(3):302-1.

14. Nicolau SM. Deficiência, gênero e práticas de saúde: estudo sobre a integralidade em atenção primária [tese]. São Paulo: Universidade de São Paulo; 2011.

15. Nicolau SM, Schraiber LB, Ayres JRCM. Mulheres com deficiência e sua dupla vulnerabilidade: contribuições para a construção da integralidade em saúde. Cien Saude Colet. 2013;18(3):872-63.

16. Gomes LF, Machado FC, Lopes MM, Oliveira RS, Medeiros-Holanda B, Silva LB, et al. Conhecimento de Libras pelos médicos do Distrito Federal e atendimento ao paciente surdo. Rev Bras Educ Med. 2017;41 (3):390-6.

17. Costa LSM, Koifman L. O ensino sobre deficiência a estudantes de medicina: o que existe no mundo? Rev Bras Educ Med. 2016;40(1):58-3.

18. Bosques G, Philip K, Francisco GE. Integration of chronic disability management in a medical student curriculum. Am J Phys Med Rehabil. 2021 Feb; 100(2S Suppl 1):S30-S33.

19. Costa LSM. Inclusão no curso médico: atenção integral à saúde das pessoas com deficiência. Rio de Janeiro: HP Comunicação; 2015.

20. Shakespeare T, lezzoni LI, Groce NE. Disability and the training of health professionals. The Lancet. 2009 Nov;374(9704):1815-6.

21. Couser GT. What disability studies has to offer Medical Education. J Med Humanit. 2010 Nov;32(1):21-30. 